\title{
Caracterização hidrológica de uma microbacia por sistema de informações geográficas (sig) objetivando identificar parâmetros pluviométricos
}

\author{
Hydrological characterization of a watershed by geographic information system (gis) aiming to \\ identify rainfall parameters \\ Caracterización hidrológica de una cuenca por sistema de información geográfica (sig) con el objetivo \\ de identificar parámetros de lluvia
}

Eliezio Nascimento Barboza ORCID: https://orcid.org/0000-0001-8100-9389

Instituto Federal do Ceará, Brasil

E-mail: eliezio1999@outlook.com

Valdemir Fonseca da Silva

ORCID: https://orcid.org/0000-0001-7871-5434 Universidade Federal da Fronteira Sul, Brasil E-mail: valsilverstone@ @otmail.com

Agílio Tomaz Marques

ORCID: https://orcid.org/0000-0001-8364-5063

Universidade Federal de Campina Grande, Brasil

E-mail: agiliotomaz@hotmail.com

Hiran Mendes Castro Filho ${ }^{1}$

ORCID: https://orcid.org/0000-0002-1418-159X

Universidad del Museo Social Argentino, Argentina E-mail: hirancastro@gmail.com

Suzana Araújo dos Santos

ORCID: https://orcid.org/0000-0001-5955-9421

Universidade Federal de Campina Grande, Brasil

E-mail: suzana.santos2007@yahoo.com.br

Matheus Matos Ferreira Silva

ORCID: https://orcid.org/0000-0002-3905-1951

Universidade Federal de Campina Grande, Brasil

E-mail: matheusmatosfs@gmail.com

Adryele Gomes Maia

ORCID: https://orcid.org/0000-0002-7433-7138 Universidade Federal do Rio Grande do Norte, Brasil

E-mail: adryelegm@gmail.com

Francisco Igor Dalles da Mata Vieira

ORCID: https://orcid.org/0000-0002-9219-9090 Instituto Federal do Ceará, Brasil

E-mail: igordm@gmail.com

\begin{abstract}
Resumo
O crescimento urbano das cidades brasileiras ocorreu de forma desordenada, sem planejamento ambiental e urbano, ocasionando diversos problemas para a população. Diversas cidades foram inicialmente ocupadas próximas aos rios e não levavam em consideração a dinâmica natural dos mesmos, ocorrendo desastrosas as enchentes e inundações pelos índices pluviométricos que proporcionam um aumento no volume dos corpos hídricos. No município do Crato não foi diferente, em que, atualmente, as áreas de escoamento da água no período de chuva encontram-se impermeabilizadas causando graves problemas para população, como fortes enchentes, processos, que privam a população de transitar com seus veículos e até mesmo a impossibilidade de tráfego dos moradores e acesso às suas residências. Dessa forma, o presente artigo objetiva definir parâmetros relacionados a distribuição espaço-temporal da precipitação e dados de vazão para a microbacia do rio Granjeiro. Sendo assim, delimitou-se a área da bacia hidrográfica do rio Granjeiro e dos seus cursos d'água, bem como aplicações de modelos estatísticos em dados pluviométricos da região e uma estimativa das intensidades e das vazões máximas pelo método racional, para determinados períodos de retorno. Verificou-se que que grande parte das precipitações máximas anuais giram em tornode 75 a $100 \mathrm{~mm}$, além de que, é observado que a distribuição de chuvas na região não necessariamente é uniforme e facilmente previsível.
\end{abstract}

Palavras-chave: Hidrologia; Inundações; Precipitação pluviométrica. 


\begin{abstract}
The urban growth of Brazilian cities occurred in a disorganized way, without urban and environmental planning, causing several problems for the population. Several cities were initially occupied near the rivers and did not take into account their natural dynamics, causing disastrous floods and inundations due to rainfall rates that provide an increase in the volume of water bodies. In the city of Crato it was no different, in that, currently, the areas of water runoff in the rainy season are impermeable causing serious problems for the population, such as heavy flooding, processes, which deprive the population of transit with their vehicles and even the impossibility of traffic for residents and access to their homes. Thus, this paper aims to define parameters related to the spatial-temporal distribution of precipitation and flow data for the micro-basin of the Granjeiro river. Thus, the watershed area of the Granjeiro river and its watercourses were delimited, as well as applications of statistical models in rainfall data of the region and an estimate of the intensities and maximum flows by the rational method, for certain return periods. It was verified that most of the maximum annual precipitation is around 75 to $100 \mathrm{~mm}$, and that the distribution of rainfall in the region is not necessarily uniform and easily predictable.
\end{abstract}

Keywords: Hydrology; Flooding; Pluviometric precipitation.

\title{
Resumen
}

El crecimiento urbano de las ciudades brasileñas ocurrió de forma desordenada, sin planificación ambiental y urbana, provocando varios problemas para la población. Varias ciudades fueron inicialmente ocupadas cerca de los ríos y no tuvieron en cuenta su dinámica natural, provocando inundaciones desastrosas e inundaciones por lluvias que proporcionan un aumento en el volumen de los cuerpos de agua. En la ciudad de Crato no fue diferente, en la cual, actualmente, las áreas de drenaje de agua en época de lluvias se encuentran impermeabilizadas provocando serios problemas a la población, como fuertes inundaciones, procesos, que privan a la población del tránsito con sus vehículos y incluso incluso la imposibilidad de circulación y acceso de los vecinos a sus viviendas. Así, el presente artículo tiene como objetivo definir parámetros relacionados con la distribución espaciotemporal de los datos de precipitación y caudal para la microcuenca del río Granjeiro. Así, se delimitó el área de la cuenca del río Granjeiro y sus cursos de agua, así como la aplicación de modelos estadísticos en datos pluviométricos de la región y una estimación de las intensidades y caudales máximos por el método racional, para determinados períodos de retorno. Se encontró que la mayor parte de las precipitaciones máximas anuales rondan los 75 a $100 \mathrm{~mm}$, además de eso, se observa que la distribución de las precipitaciones en la región no es necesariamente uniforme y fácilmente predecible.

Palabras clave: Hidrología; Inundaciones; Lluvia.

\section{Introdução}

De acordo com Tucci (2001), a Hidrologia se refere ao estudo da água, bem como sua ocorrência, percolação e disposição, propriedades físico-química. A hidrologia urbana, por sua vez, estuda as zonas urbanas que, em sua maioria, detém a superfície do solo impermeabilizada e faz a análise do impacto ocasionado pelo desenvolvimento das cidades com relação ao ciclo das águas e os meios para combater os efeitos negativos a partir do estudo do escoamento superficial (Martins, 2010).

Em análises hidrológicas, destaca-se os estudos na bacia hidrográfica. Para Araújo et al. (2009, p.1), “a bacia hidrográfica é o elemento fundamental de análise no ciclo hidrológico, principalmente na sua fase terrestre, que engloba a infiltração e o escoamento superficial”. Em uma bacia hidrográfica, suas características físicas assumem um importante papel, tendo em vista que no ciclo hidrológico podem influenciar no processo de infiltração, evapotranspiração e escoamento, seja superficial ou subsuperficial (Tonello et al., 2006). Logo, destaca-se a importância da delimitação da bacia em estudos hidrológicos, objetivando as análises morfométrica por meio de parâmetros como: densidade de drenagem, formas da bacia, fator de forma (kf), Coeficiente de Compacidade (kc), índice de circularidade e entre outros.

Neste sentido, destaca-se os estudos hidrológicos em bacias hidrográficas. A bacia de interesse deste estudo é a do rio Granjeiro, em que é o rio principal da microbacia da sub-bacia hidrográfica do rio Salgado, no qual drena parte do município do Crato/ CE e é afluente do rio Jaguaribe. Ele nasce na encosta da Chapada do Araripe (com declividade elevada, >70\%) e possui talvegue de 8,0 a $0,1 \%$ (Lima Neto, 2008). A microbacia do rio Granjeiro abrange quase a totalidade do município de Crato e suas características de ocupação combinadas com relevos do entorno oeste da Chapada do Araripe e regimes pluviométricos superiores aos do semiárido favorecem a geração de inundações (Paiva, 2016).

Sendo assim, a presente pesquisa justifica-se pela necessidade de estudo de ordem hidrológica na bacia, visto que as áreas de escoamento da água no período de chuva encontram-se impermeabilizadas causando graves problemas para população, 
como fortes enchentes, processos erosivos ativos, que privam a população de transitar com seus veículos e até mesmo a impossibilidade de tráfego dos moradores e acesso às suas residências.

Dessa forma, torna-se necessário um estudo para quantificar o escoamento superficial gerado na área em estudo, através de modelagem chuva-vazão, para definir medidas eficientes na solução dos problemas causados pela falta de drenagem urbana. Neste sentido, o presente artigo objetiva definir parâmetros relacionados a distribuição espaço-temporal da precipitação e dados de vazão para a microbacia do rio Granjeiro.

\section{Metodologia}

\subsection{Caracterização da área de estudo}

A microbacia de interesse nesse estudo é a do Rio Granjeiro, no qual está localizada no município do Crato (Figura 1), mais especificamente naMetropolitana do Cariri (RMC). A sede do município do Crato apresenta como coordenadas geográficas 7¹4'03" de Latitude (S) e 39²4’34” de Longitude (W) (Pinheiro \& Silva, 2019). A Figura 1 apresenta a localização do subdistrito em relação ao município.

Figura 1: Mapa de localização do subdistrito de Crato, Ceará.

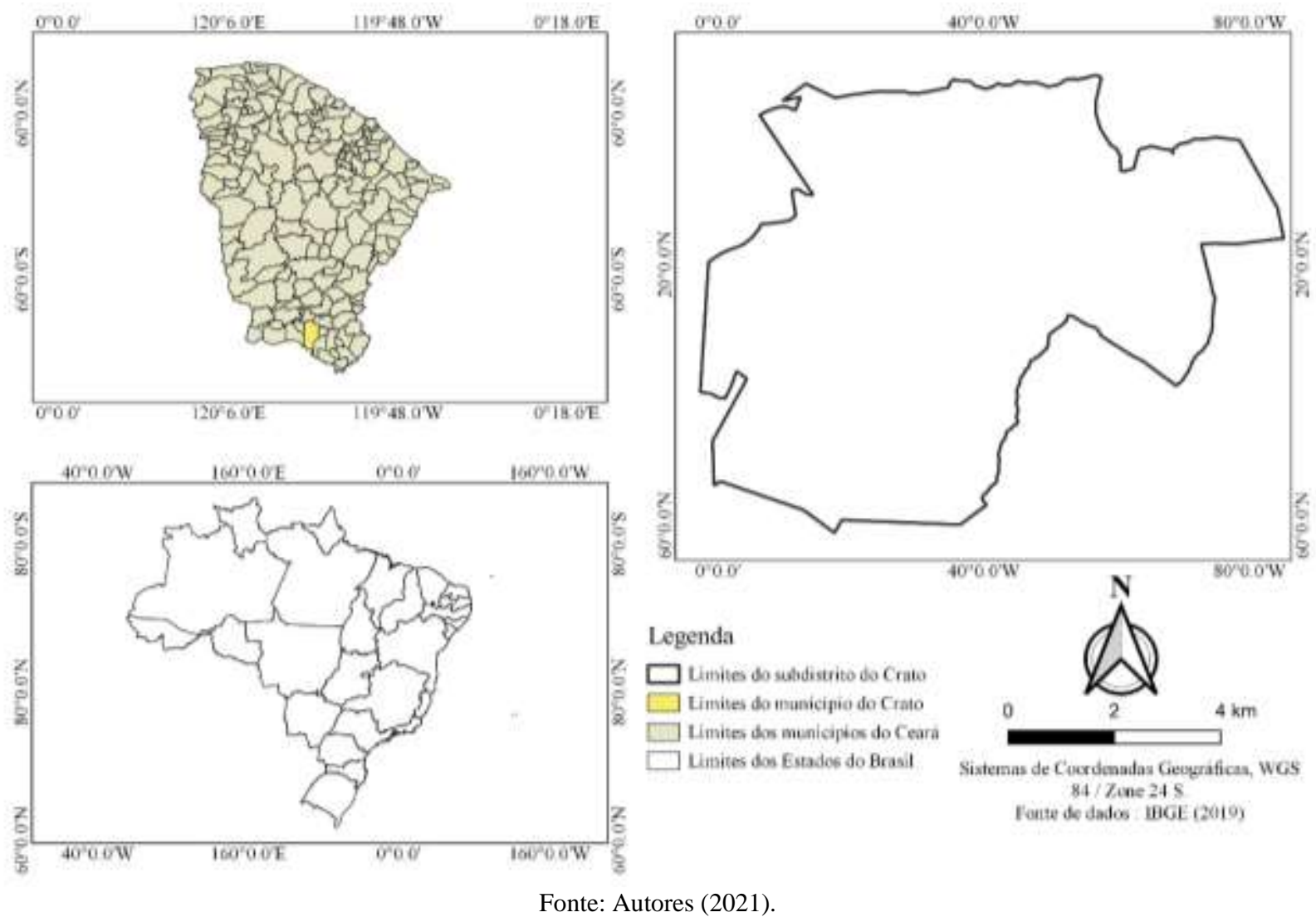

A Figura 1 representa a localização do subdistrito Crato em relação ao munícipio de Crato, Ceará. O munícipio limitase ao norte com Caririaçu e Farias Brito, ao sul com o Estado de Pernambuco e Barbalha, a Leste com Barbalha, Juazeiro do Norte e Caririaçu e a oeste com Nova Olinda, Santana do Cariri e o Estado de Pernambuco (IPECE, 2015).

No município, destaca-se os aspectos geomorfológicos da chapada do Araripe na paisagem nordestina, possuindo mais de $180 \mathrm{~km}$ no seu eixo maior e $30 \mathrm{~km}$ de largura (Guerra \& Sampaio, 1996). A presença da Chapada do Araripe distingue o Crato de outras cidades nordestinas pela diferença climática pela influência direta do efeito orográfico da Chapada, integran do um topo clima (Lima, 2015). 
O sistema hídrico da região é constituído por mananciais de superfície, poços profundos, particulares e nascentes. Os depósitos nessa região ligados aos sedimentos da Bacia Sedimentar do Araripe, recobrindo o embasamento Pré-Cambriano Gnaíssico-migmatítico da Província Borborema (Lima, 2015).

O município de Crato apresenta um relevo dissecado pelos rios que drenam o município, e pela ação das águas pluviais que também têm o seu papel no modelado do relevo (Guerra \& Sampaio, 1996). A topografia de Crato é acidentada e o relevo varia de 399 a $702 \mathrm{~m}$ de altitude. No Oeste, Sudoeste e Sul as altitudes apresentaram-se como mais elevadas, nas demais áreas as altitudes são menores.

O município está inserido a microbacia do Rio Granjeiro sendo favorecido pela ocorrência de chuvas orográficas, com valores de precipitações pluviométricas mais elevadas do que outros municípios do Cariri (Silva, 2015). O período chuvoso da região é de janeiro a abril, com valores superiores à média, já o período de seca é de maio a dezembro, com valores a baixos da média de precipitação pluviométrica, exceto em dezembro. O mês de maior precipitação é em março com média de $258.3 \mathrm{~mm}$ e o mês com menor quantidade de precipitação pluviométrica é em agosto, com média de $2.5 \mathrm{~mm}$ no mês. O período de seca no município de extremamente intenso, com cinco meses do ano no qual a precipitação é basicamente inexistente.

\subsection{Procedimentos metodológicos}

Primeiramente realizou-se a delimitação da bacia hidrográfica, no qual utilizando-se de um modelo digital de elevação (MDE), que é oriundo da imagem SRTM, na qual foi possível obter por meio do projeto TOPODATA - INPE, o qual possui uma resolução espacial de 30 metros. A região de interesse está presente na carta SB-24-Y-D, com centro de latitude $7^{\circ} 27^{\prime}$ S e longitude $39^{\circ} 45^{\prime} \mathrm{W}$.

Para processamento e a criação de modelos hidrológicos precisos, utilizou-se o software ArcGIS®. Inicialmente foi realizado o georreferenciamento dos dados MDE, utilizando um sistema de coordenadas planas, para que o software possa identificar a localização das camadas seguintes. Na ferramenta Arc hydro, o software irá analisar os dados altimétricos do raster MDE e a partir da rede de drenagem, delimitar as microbacias da área.

Entre os plugins do software, utilizou-se a ferramenta fill skins, as ferramentas flow Direction e flow acumulation, as ferramentas stream definition e stream segmentation, catchment grid delineation e a ferramenta catchment polygon processing. Por último, com o adjoint catchment processing, foram definidas as microbacias com a sua rede própria de drenagem em formato vetor, pronto para a delimitação individual dasbacias. Através da ferramenta drainage point processing, foi apresentado os pontos de drenagemde cada bacia, e a ferramenta batch point Generation, foi definido o ponto de drenagem da área de interesse. Por último, com o watershed delineation, a(s) microbacia(s) são delimitadas e prontaspara a análise de forma individual, apresentando todos os cursos d'água.

\subsubsection{Características física da bacia}

O comprimento da linha de maior profundidade no leito de um rio foi extraído no software ArcGIS, através de ferramentas de medição. Já a declividade (i) foi calculada pela Equação 1, no qual relaciona o desnível altimétrico do terreno $(\Delta \mathrm{h})$ em $\mathrm{m}$, com a extensão do rio (L) também em m.

$i=\frac{\Delta h}{L}(1)$

O tempo de concentração, por sua vez, é a duração em que o fluido fica retido na superfície (Queiroz \& Alves, 2020). Neste estudo, utilizou-se a Equação de Picking, que relaciona o comprimento do talvegue (Lt) em km e a declividade (i) em $\mathrm{m} / \mathrm{m}$ (Equação 2). 
$T_{c}=57\left(\frac{L^{2}}{I}\right)^{0,38}$

A precipitação histórica foi obtida através dos postos pluviométricos da região, no qual são disponibilizados pela Fundação Cearense de Meteorologia e Recursos Hídricos (FUNCEME). O preenchimento das falhas de dados pluviométricos foi realizado através de regressão linear simples, no qual foi aplicadocorrelacionando as precipitações do posto com falhas, com dados de um posto vizinho, atentando-se para que os dados analisados referenciem um período comum de tempo.

Após o preenchimento das falhas, foi necessário verificar a homogeneidade dos dados obtidos. Essa etapa verifica mudanças na série temporal, utilizando o diagrama de dupla massa.Esse método consiste em acumular os valores diários do posto analisado de forma mensal, e compará-lo com a média acumulada mensal dos postos vizinhos, fazendo assim uma comparação regional dos dados de chuva (Bertoni \& Tucci, 2001).

Realizou-se a análise de frequência dos dados de precipitação considerando a série totalde dados, série parcial ou série anual, onde foi verificado as precipitações máximas ao longo das séries anuais e feito uma distribuição de frequência, seguindo modelos probabilísticos. Posteriormente, foi é necessário dividir esses valores em intervalos, e em cada um desses intervalos é medido uma frequência com que essas precipitações ocorreram.

Foi realizada uma classificação dos valores, do maior para o menor valor, atribuindo o valor de m para sua ordem na classificação e n para a quantidade de anos a quão estão sendo analisados, para que assim, possam ser aplicados (Equação 3 ).

$F=\frac{m}{n}(3)$

Necessitou-se verificar a adequabilidade ao modelo probabilístico. Logo, foi utilizado o teste de aderência Quiquadrado, onde K é referente aos resultados possíveis, Ei corresponde aos resultados ocorridos e Oi aos resultados esperados (Equação 4).

$x^{2}=\sum_{i=1}^{n} \frac{\left(o_{i}-e_{i}\right)^{2}}{e_{i}}(4)$

A curva IDF, por sua vez, calculada de acordo com a Equação 5, em que $i$ representa a intensidade da chuva em $\mathrm{mm} / \mathrm{min}$, Tr otempo de retorno, em anos, $\mathrm{t}$ a duração da chuva em min, e a, b, c, s e n são parâmetros específicos determinados para cada local.

$i=\frac{a \cdot T_{r}^{b}}{(t+c)^{d}}$

Como o município do Crato não dispõe de pluviógrafos, Sobrinho (2011) estimou os coeficientes da Equação 5 para o município de Crato, através de dados pluviométricos, reproduzindo resultados confiáveis (Tabela 1). 
Tabela 1: Parâmetros da equação de chuvas intensas para o município de Crato-CE.

\begin{tabular}{cccccc}
\hline Variável & $\mathbf{a}$ & $\mathbf{b}$ & $\mathbf{c}$ & $\mathbf{N}$ & $\mathbf{s}$ \\
\hline Crato & 20,24 & 0,0769 & 5,45 & 0,723 & $-2,99$
\end{tabular}

Fonte: Adaptado de Sobrinho (2011).

Para estimar a vazão de projeto, empregou-se o método racional, em que considera a duração da precipitação intensaigual ao tempo de concentração da bacia, adota um coeficiente único de perdas denominado C,não avalia o volume da cheia e a distribuição temporal das vazões (Equação 6), em que C é coeficiente de perda (obtido através Tucci (2001) apud ASCE (1969); I é a intensidade da precipitação em $\mathrm{mm} / \mathrm{min}$; A é a área da bacia em $\mathrm{km}^{2}, \mathrm{~K}$ é o fator de correlação (para as unidades citadas, Q a vazão em m³/s;

$Q=\frac{C \cdot I \cdot A}{K}(6)$

\section{Resultados e Discussões}

Delimitou-se a bacia hidrográfica, bem como os rios principais e secundários. A área delimitada foi de 1,75\% da área total do município, no entanto, está situado em uma área de grande concentração urbana, com comércios e áreas residenciais, necessitando um estudo hidrológico para a região, com potencial de desenvolvimento. A área possui 9 microbacias em sua constituição, além de um rio principal e 4 rios secundários, como mostra a Figura 2.

Figura 2: Delimitação da bacia.

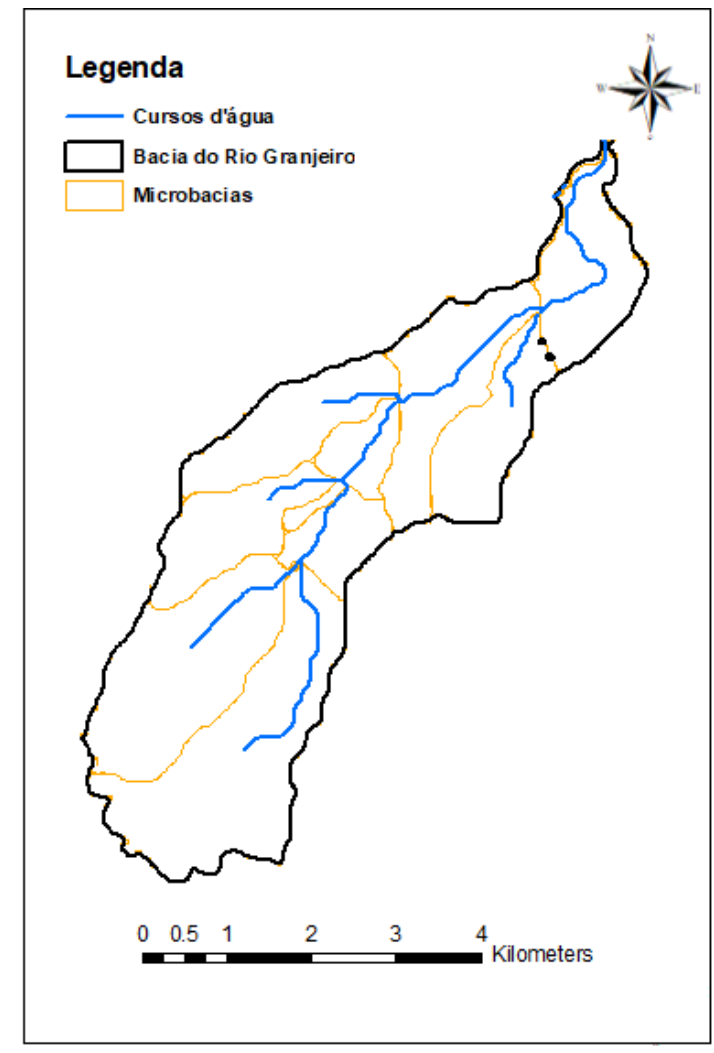

Fonte: Autores (2021).

Com a delimitação, foi extraído parâmetros geométricos para a definição do tempo deconcentração (Tc), conforme 
mostrado na Tabela 2. Através desses dados, foi possível calcular o tempo de concentração da bacia hidrográfica do rio granjeiro, resultando em um valor de 78,3 $\mathrm{min}$.

Tabela 2: Dados da microbacia.

\begin{tabular}{cccc}
\hline Variável & Siglas & Dados & Unidade \\
\hline \hline Área total & $\mathrm{A}$ & 19,87 & $\mathrm{~km}^{2}$ \\
Comprimento do talvegue & $\mathrm{Lt}$ & 7,67 & $\mathrm{~km}$ \\
Declividade média do curso d'água principal & $\mathrm{Dp}$ & 2,47 & $\%$ \\
\hline
\end{tabular}

Fonte: Autores (2021).

Para a análise pluviométrica da bacia foi escolhido o posto de Crato, localizado na região central da área de estudo. A sua escolha se justifica pela sua localização e por possuir um grande número de séries pluviométricas sem interrupções. Na etapa de análise de dados pluviométricos, foi necessário o preenchimento de falhas do posto pluviométrico do Crato. Paraisso, utilizou-se como referência os dados do posto localizado em Juazeiro do Norte a uma distância de aproximadamente $13 \mathrm{~km}$. O posto foi escolhido por possuir um número baixo de falhas e uma série histórica semelhante ao posto influente da bacia, além de ser um dos mais próximos ao posto a ser analisado.

Os dados do posto do Crato apresentam falhas consideráveis, principalmente no ano de 2012. Assim, foi considerado o período anterior a essas falhas para a plotagem do gráfico dedispersão mostrado na Figura 7. Foi necessário o ajuste com a exclusão de valores muito distantes da linha de tendência, para que assim possa haver um melhor desempenho na análise.Com o auxílio do software Microsoft Excel, e utilizando de regressão linear simples, chegou- se ao gráfico mostrado na Figura 3.

Figura 3: Preenchimento de Falhas.

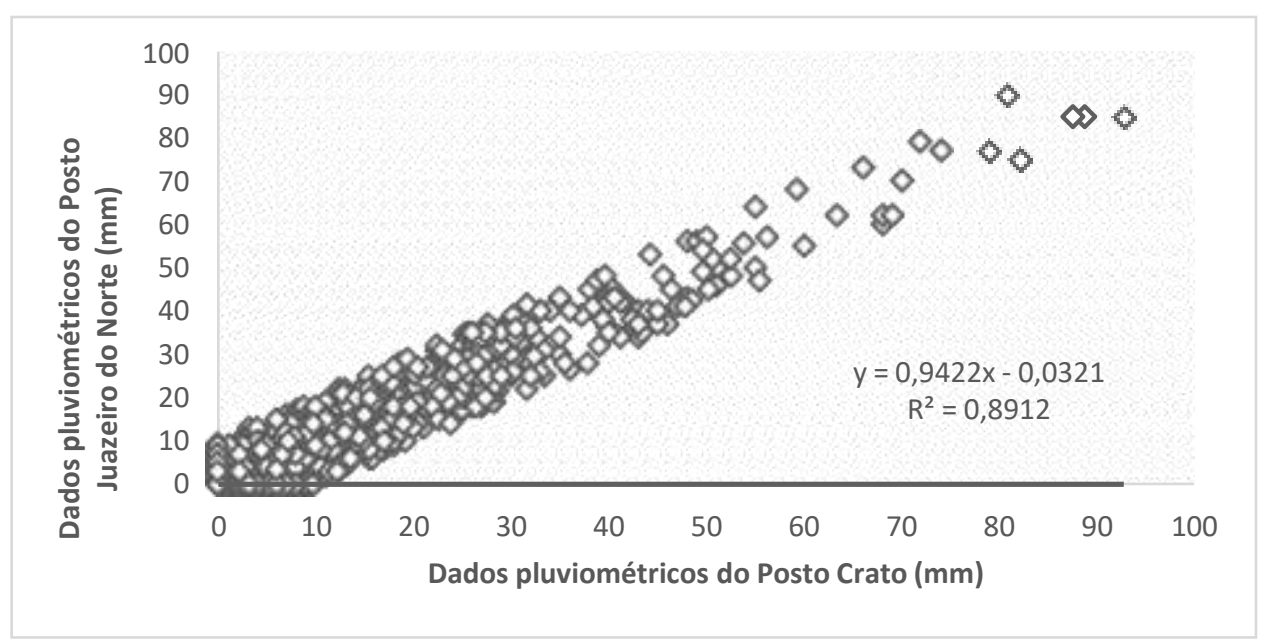

Fonte: Autores (2021).

$\mathrm{O}$ valor de $\mathrm{R}^{2}$ apresentado no gráfico mostra o quão próximos os valores estão da linhaajustada, nesse caso cerca de $89,12 \%$. Com a equação $\mathbf{y}$, é possível chegar ao valor referente afalha diária do posto analisado, utilizando o parâmetro x, referente ao dado registrado de um posto próximo, neste caso, localizado em Juazeiro do Norte. Para a análise de consistência dos dados do posto pluviométrico de Crato, foi acumulado os dados de forma mensal, da mesma forma para o posto pluviométrico de Juazeiro do Norte, que será usado como base de comparação. Após isso, utilizando o software MicrosoftExcel, gerado o gráfico 
mostrado na Figura 4.

Figura 4: Análise de consistência.

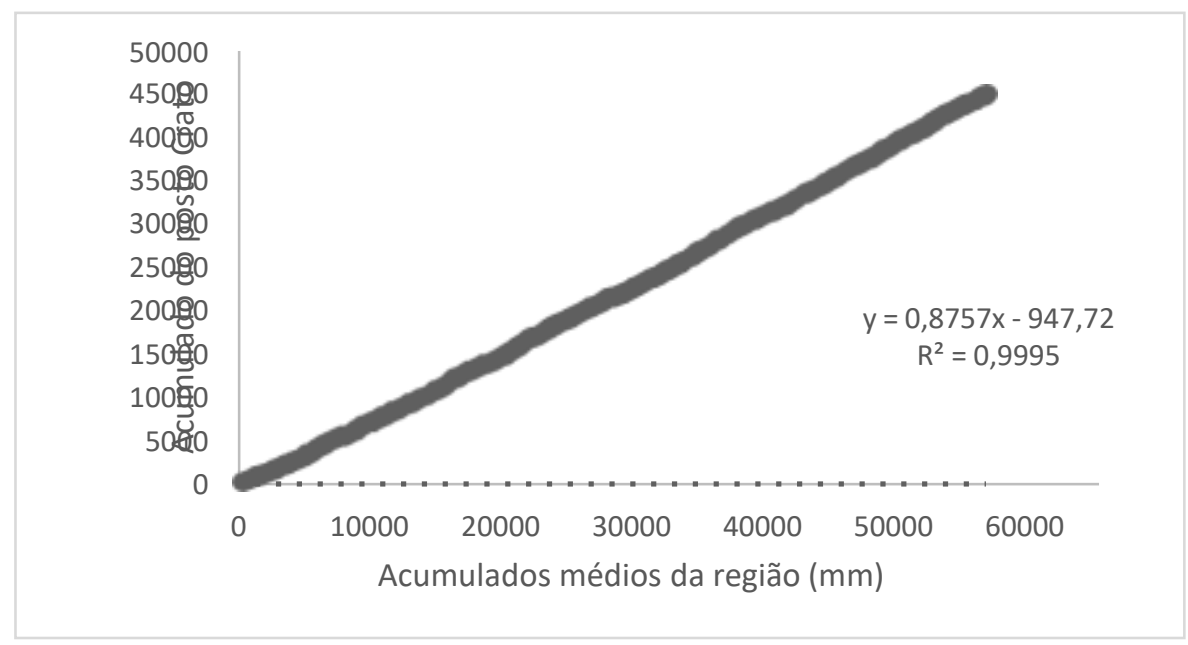

Fonte: Autores (2021).

A Figura 4, com $\mathrm{R}^{2}=0,99$, mostra uma linearidade dos dados do posto analisadoem relação aos postos circunvizinhos, sem uma visível alteração de inclinação. Logo, é possívelafirmar que os dados do posto em questão não possuem inconsistências e podem ser considerados homogêneos.

Além disso, também utilizando os dados, foi possível gerar o gráfico mostrado na Figura 5, que mostra a precipitação anual registrada no posto pluviométrico, além da precipitação anual média registrada, durante a série temporal de 1974 a 2020.

Figura 5: Valores de precipitação (ano).

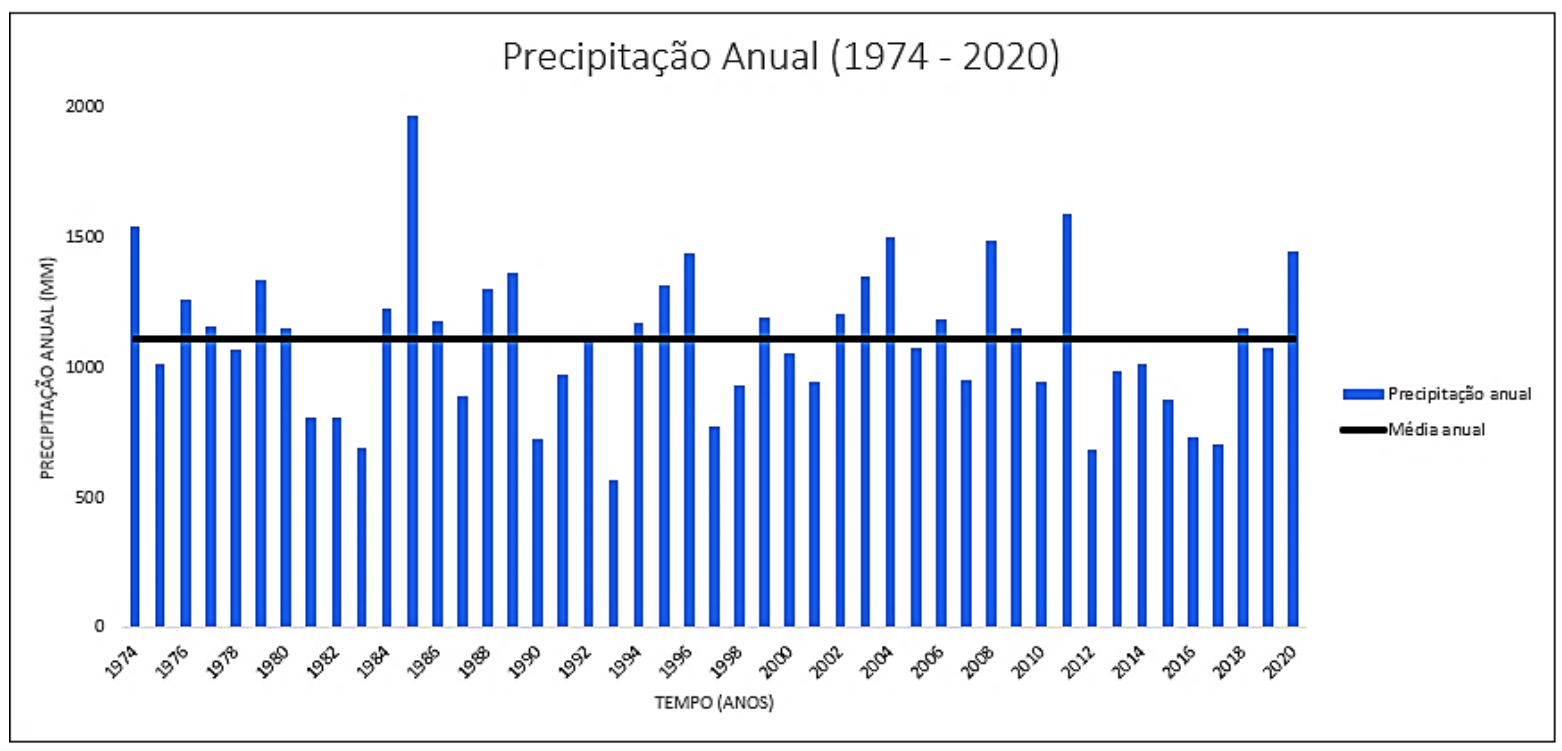

Fonte: Autores (2021).

Além disso, verificou-se as precipitações médias diárias máximas anuais na bacia, quegiram em torno de 99,04 mm. Valor esse que em aproximadamente $53,20 \%$ dos anos, não é alcançado, porém, existem casos excepcionais, como no ano de 2011, onde foi registrado precipitações de até $162 \mathrm{~mm}$

$\mathrm{Na}$ etapa de distribuição de frequência, dividiu-se as precipitações máximas emintervalos de $25 \mathrm{~mm}$ de chuva, 
gerando então sete classes de 0 à $175 \mathrm{~mm}$, considerando fi comocomo a frequência observada, equivalente a quantidade de vezes que os valores de precipitaçãomáxima correspondente aos intervalos de limite se repetem, Fe a frequência esperada, calculadacom base na distribuição gama realizada, utilizando os coeficientes. Os valores do teste de Qui-quadrado foram: $\mathrm{A}=0,04 ; \alpha=13,64$; $\beta=7,39 ; \Gamma(\alpha)=2452810442,54$. Os parâmetros de distribuição de frequência estão apresentados na Tabela 3.

Tabela 3: Parâmetros de distribuição de frequência.

\begin{tabular}{|c|c|c|c|c|c|c|c|c|c|c|}
\hline $\begin{array}{l}\mathrm{N}^{0} \text { da } \\
\text { Classe }\end{array}$ & $\begin{array}{c}\text { Limite } \\
\text { Inferior }\end{array}$ & $\begin{array}{c}\text { Limite } \\
\text { Superior }\end{array}$ & $\mathbf{F i}$ & $\mathbf{x i}$ & fi.xi & $\ln (x)$ & $\ln (x) . f i$ & $\begin{array}{c}\text { Dist. } \\
\text { Gama } \\
\text { Acum. }\end{array}$ & $\mathbf{F e}$ & $\begin{array}{c}\text { Qui- } \\
\text { Quadrado }\end{array}$ \\
\hline 1 & "0 & 25 & 0 & 12,5 & 0 & 2,53 & 0,00 & $2,18 \mathrm{E}-05$ & 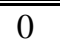 & 0,001 \\
\hline 2 & 25 & 50 & 0 & 37,5 & 0 & 3,62 & 0,00 & $1,31 \mathrm{E}-02$ & 1 & 0,613 \\
\hline 3 & 50 & 75 & 8 & 62,5 & 500 & 4,14 & 33,08 & $1,72 \mathrm{E}-01$ & 7 & 0,041 \\
\hline 4 & 75 & 100 & 18 & 87,5 & 1575 & 4,47 & 80,49 & $5,24 \mathrm{E}-01$ & 17 & 0,121 \\
\hline 5 & 100 & 125 & 11 & 112,5 & 1237,5 & 4,72 & 51,95 & $8,19 \mathrm{E}-01$ & 14 & 0,592 \\
\hline 6 & 125 & 150 & 8 & 137,5 & 1100 & 4,92 & 39,39 & $9,52 \mathrm{E}-01$ & 6 & 0,517 \\
\hline 7 & 150 & 175 & 2 & 162,5 & 325 & 5,09 & 10,18 & $9,90 \mathrm{E}-01$ & 2 & 0,019 \\
\hline $\bar{\Sigma}$ & & & 47 & & 4737,5 & & 215,094 & & & 1,905 \\
\hline
\end{tabular}

Fonte: Autores (2021).

Com esses parâmetros, obteve-se o grau de liberdade igual a 6, e um intervalo de confiança adotado de 99,5\%, onde utilizando a tabela de Qui-quadrado de Pearson e Hartley (1966), obtêm-se um Qui quadrado tabelado de 16,7, superior ao calculado, o que mostra que adistribuição gama utilizada é compatível com a análise. Assim, é possível traçar o gráfico de distribuição de frequência observada e a frequência esperada, como é mostrado na Figura 6.

Figura 6: Distribuição de frequência de chuvas observada e esperada.

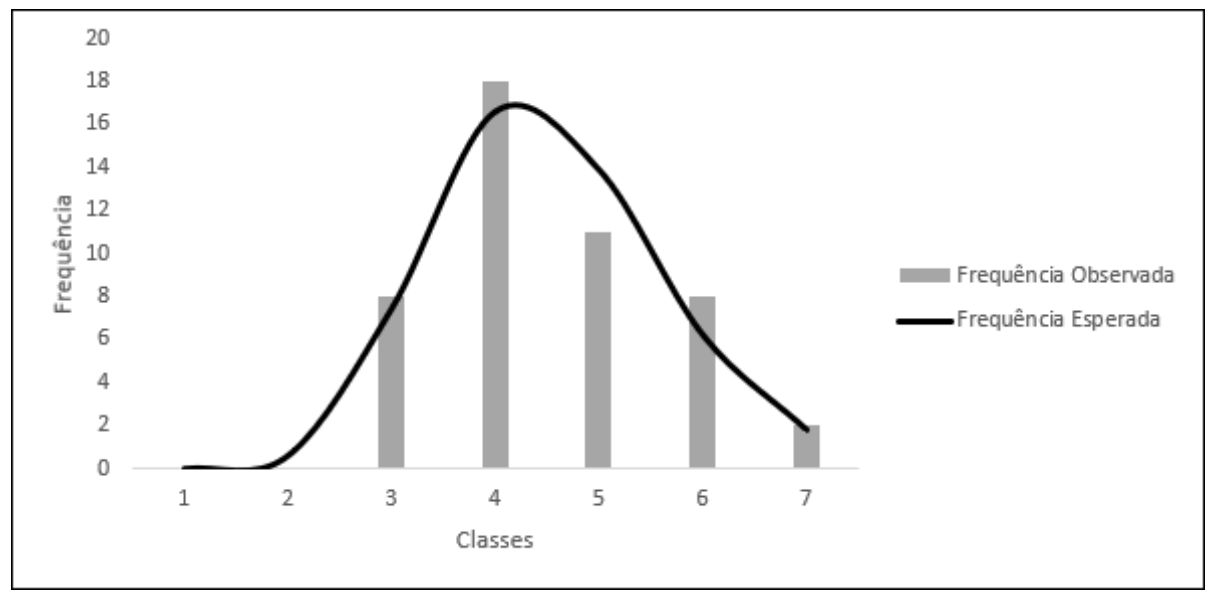

Fonte: Autores (2021).

No que se refere o cálculo de intensidade de chuvas, o tempo de duração da precipitação variou de 0 a 240 min e o tempo de retorno estabelecido foi de 5, 10, 25,50 e 100 anos. Assim, foi possível traçar o gráfico com as intensidades de chuva no eixo da ordenada e tempo de duração de chuva no eixo da abscissa, onde cada curva representa o tempode retorno utilizado, conforme a Figura 7. 
Research, Society and Development, v. 11, n. 2, e39411225490, 2022

(CC BY 4.0) | ISSN 2525-3409 | DOI: http://dx.doi.org/10.33448/rsd-v11i2.25490

Figura 7: Intensidades calculadas pela equação IDF segundo Sobrinho (2011)

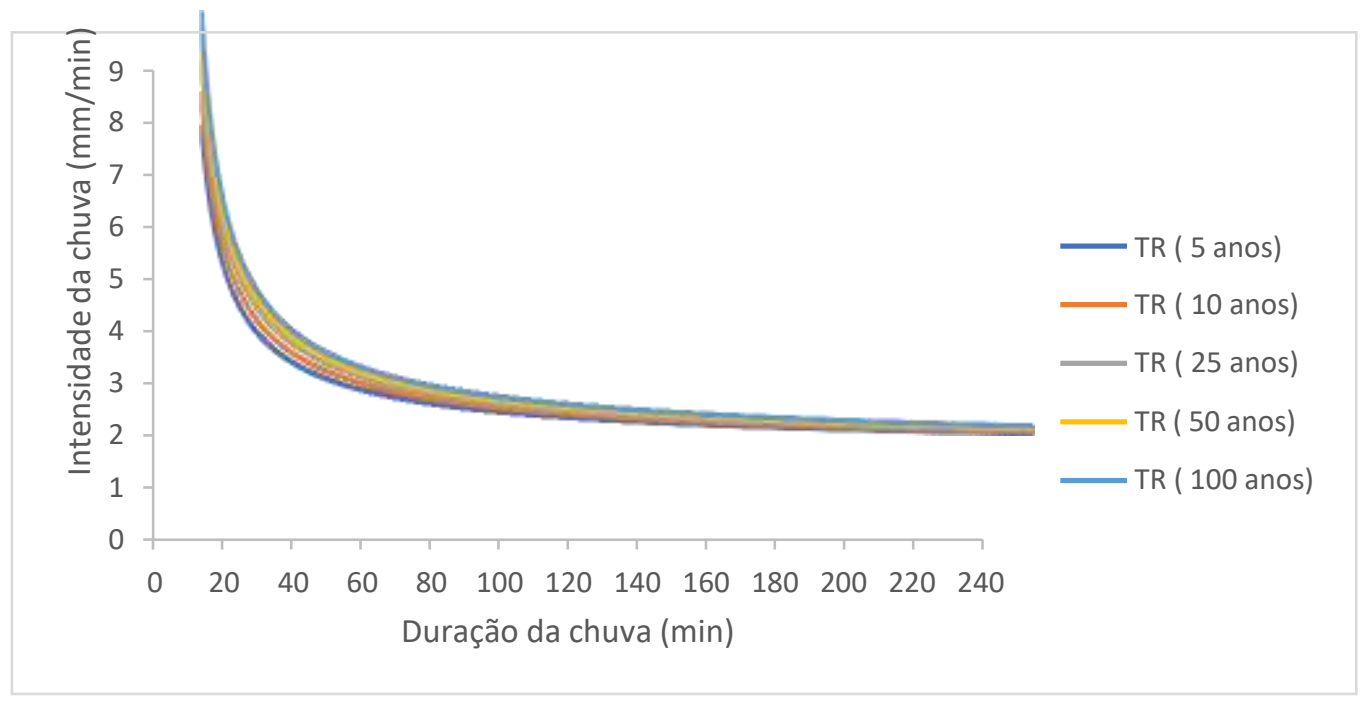

Fonte: Autores (2021).

Com isso, foi possível estimar as vazões utilizando o método racional. Foi considerando um valor de 0,88 por se tratar de uma microbacia urbana, onde a parte mais sucessível a enchentes é coberta por concreto. Assim, calculou-se as vazões utilizando dados de intensidade mostrados na Figura 7, é gerado o gráficoque apresenta os valores de vazão correspondente a intensidade, para cada período de retorno, como é mostrado nas Figura 8.

Figura 8: Gráfico de vazão e intensidade.

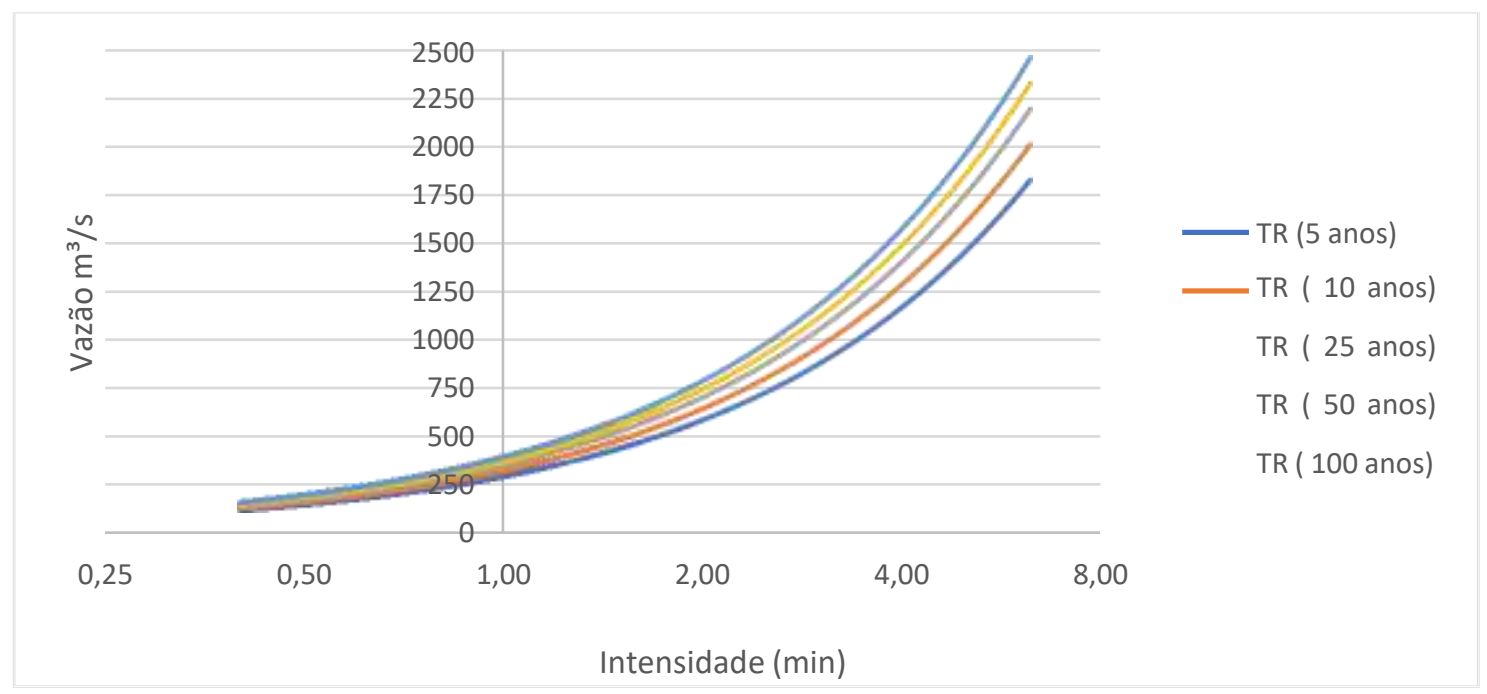

Fonte: Autores (2021).

Com os gráficos acima, foi possível estimar a vazão atuante na microbacia para chuvas de duração inferior a 240 min e intensidades calculadas para tempos de retorno de 5, 10, 25, 50 e 100 anos, podendo ser utilizado para aplicações práticas em processosde dimensionamento de estruturas drenagem. A Tabela 4 apresenta o cálculo das vazões utilizando a duração da chuva igual à do tempo de concentração da bacia. 
Research, Society and Development, v. 11, n. 2, e39411225490, 2022

(CC BY 4.0) | ISSN 2525-3409 | DOI: http://dx.doi.org/10.33448/rsd-v11i2.25490

Tabela 4: Estimativa das vazões pelo método racional.

\begin{tabular}{cccccc}
\hline \hline $\operatorname{Tr}(\mathbf{a n o s})$ & $\mathbf{t}(\mathbf{m i n})$ & $\mathbf{i}(\mathbf{m m} / \mathbf{m i n})$ & $\mathbf{C}$ & $\mathbf{A ~ ( \mathbf { k m } ^ { 2 } )}$ & $\mathbf{Q}\left(\mathbf{m}^{\mathbf{3}} / \mathbf{s}\right)$ \\
\hline \hline 5 & & 0,87 & & & 252,37 \\
10 & 0,95 & & & 278,36 \\
25 & 78,3 & 1,05 & 0,88 & 19,87 & 305,60 \\
50 & & 1,12 & & & 325,33 \\
100 & & 1,18 & & & 345,43 \\
\hline \hline
\end{tabular}

Fonte: Autores (2021).

Sendo assim, a Tabela 4 mostra os valores para diferentes tempos de retorno, em que variou de 252,37 para 5 anos até 345,43 , para 10 anos.

\section{Considerações Finais}

A partir deste estudo, foi possível verificar que grande parte das precipitações máximas anuais giram em tornode 75 a 100 mm, além de que, é observado que a distribuição de chuvas na região não necessariamente é uniforme e facilmente previsível, podendo ocorrer precipitações com valoresmuito acima da média em séries não muito chuvosas, como ocorrido nos anos de 1992 , 2004 e 2015.

A partir dos dados encontrados, verificou-se que os dados são aceitáveis quando comparados a estudos anteriores, além de contribuir com uma base atualizada de dadospluviométricos, resultando em uma maior precisão para estudos posteriores. Considerando ainda, que dados de intensidade de chuva e vazão gerados podem servir como base de estudose para a aplicação de outros métodos de distribuição ao longo do tempo, ou ainda, como parâmetro para projetos de drenagem urbana na região, podendo assim beneficiar a população e minimizar os impactos causados pelas cheias.

\section{Referências}

Araújo, L. E. et al. (2009). Bacias hidrográficas e impactos ambientais. Qualitas Revista Eletrônica, 8(1).

Basso, Raviel Eurico et al. (2016). Revisão das isozonas de chuvas intensas do Brasil. Engenharia Sanitária e Ambiental, 21, 635-641.

Bertoni, J. C., Tucci, C. E. M. (1993). Precipitação. Hidrologia: ciência e aplicação, 2, 177-242.

Costa Saraiva, A. L. B. da., Do Vale, C. C., \& Zanella, M. E. (2017). Comportamento dos Elementos Climáticos no Município de Mossoró (RN) e os Impactos na Saúde Humana. Revista GeoInterações, 1(1), 87-105.

IPECE. Instituto de Pesquisa e Estratégia Econômica do Ceará. (2015). Perfil e Municipal. http://www.ipece.ce.gov.br

Lima Neto, R. T. et al. (2008). Análise morfométrica e ambiental da microbacia hidrográfica do rio Granjeiro, Crato/CE. Rem: Revista Escola de Minas, 61, 365-369.

Martins, C. M. T. et al. (2010). Hidrologia urbana: conceitos básicos. Série Cursos Técnicos, n. 1.

Moreira, A. A. C. (2013). Modelagem hidrológica da bacia hidrográfica do Rio Granjeiro - Crato-CE: composição do cenário atual e simulações de uso e ocupação do solo. 2013. 163 f. Dissertação (Mestrado em Engenharia Civil: Recursos Hídricos) - Centro de Tecnologia, Universidade Federal do Ceará, Fortaleza, 2013.

Naghettini, M., \& Pinto, É. J. de A. (2007). Hidrologia estatística. CPRM.

Pfafstetter, O. (1957). Chuvas intensas no Brasil: relação entre precipitação, duração e frequência de chuvas em 98 postos com pluviógrafos. Rio de Janeiro: DNOCS.

Pinheiro, M. A. et al. (2015). Análise e Classificação das Formas de Uso e Ocupação doSolo da Microbacia do Rio Granjeiro, Crato/CE. In: Congresso Brasileiro de ciência do Solo, 35(1), 1-4.

Shams, J. C. A., Giacomeli, D. C., \& Sucomine, N. M. (2009). Emprego da arborização na melhoria do conforto térmico nos espaços livres públicos. Revista da Sociedade Brasileira de Arborização Urbana, 4(4), 01-16.

Silva Brito, D., \& Silva, J. M. O. (2012). Estudo dos impactos pluviométricos e os eventos extremos no município de Crato-CE. Revista Geonorte, 3(8), 964- 
Research, Society and Development, v. 11, n. 2, e39411225490, 2022

(CC BY 4.0) | ISSN 2525-3409 | DOI: http://dx.doi.org/10.33448/rsd-v11i2.25490

976-964-976.

Silva, J. M. O. (2015). Uso do Sensoriamento Remoto para a estimativa da temperatura de superfície na Microbacia do Rio Granjeiro/Crato-Ceará. Geosaberes: Revista de Estudos Geoeducacionais, 6(2), 130-144.

Silveira, A. L. L. da. (2005). Desempenho de Fórmulas de Tempo de Concentração emBacias Urbanas e Rurais. RBRH - Revista Brasileira de Recursos Hídricos, $10(1), 5-23$

Sobrinho, V. F. (2011). Aplicação do método das isozonas na obtenção das equações IDF de chuvas intensas dos municípios de Juazeiro do Norte, Barbalha e Crato - CE. 72 f. Dissertação (Mestrado em Engenharia Civil) - Centro de Tecnologia, Universidade Federal do Ceará, Fortaleza, 2011.

Tibúrcio, E. C., \& Castro, M, A. H. de. (2007). Uso de Sistema de Informação Geográfica para Determinação de Vazões Máximas em Projetos de Hidrologia. In: Simpósio Brasileiro de Recursos Hídricos, 17(1), 1-20.

Tonello, K. C. et al. (2006). Morfometria da bacia hidrográfica da Cachoeira das Pombas, Guanhães-MG. Revista Árvore, $30(5), 849-857$.

Tonello, K. C. (2005). Análise hidroambiental da bacia hidrográfica da cachoeira das Pombas, Guanhães, MG. Tese (Doutorado em Ciências Florestal) UniversidadeFederal de Viçosa.

Torrico, J. J. T. (1974). Práticas hidrológicas. Transcon.

Tucci, C. E. M. (2001). Hidrologia Ciência e Aplicação. (2a ed.), Editora da UFRGS.

Tucci, C. E. M. (1993). Água no meio urbano. Livro água doce, 1-40.

Tucci, C. E. M. (2003). Drenagem urbana. Ciência e cultura, 55(4), 36-37.

Villela, S. M., \& Mattos, A. (1975). Hidrologia aplicada. McGraw-Hill doBrasil. 